\title{
Estructura de una población del árbol Peltogyne purpurea (Cesalpinaceae) en un bosque intervenido de la Península de Osa, Costa Rica
}

\author{
Braulio Vílchez ${ }^{1} \&$ Oscar Rocha ${ }^{2,3}$ \\ Instituto Tecnológico de Costa Rica, Escuela de Ingeniería Forestal; bvilchez@itcr.ac.cr \\ Universidad de Costa Rica, Escuela de Biología; ojrocha@cariari.ucr.ac.cr \\ Kent State University, Department of Biological Sciences, Kent, Ohio, 44242; orocha@kent.edu
}

Recibido 27-III-2003. Corregido 28-VI-2004. Aceptado 08-II-2005.

\begin{abstract}
Population structure of the tree Peltogyne purpurea (Cesalpinaceae) in an altered forest at Osa Peninsula, Costa Rica. The regeneration of the rain forest tree Peltogyne purpurea Pittier after selective logging was studied in Mogos, Osa Península, Costa Rica. The distribution of all adult trees of P. purpurea according to diameter at breath height ( $\mathrm{dbh}$ ) categories revealed that the distribution of the adult trees followed a bell-shaped curve. The largest number of individuals was found in the 70 to $80 \mathrm{~cm}$ dbh category. We did not find any significant differences in the distribution of saplings around seed-producing trees. There were similar numbers of saplings in the four transects established around each reproductive tree following the four cardinal directions. Overall, the majority of the saplings were found in the first meters around each seed-producing tree. There was another peak in the number of saplings at the distance where the crown of the tree ended. Logging can negatively affect the regeneration of P. purpurea. Rev. Biol. Trop. 54 (3): 1019-1029. Epub 2006 Sept. 29.
\end{abstract}

Key words: Peltogyne purpurea, tropical trees, population structure, forest regeneration, population dynamics, sapling survival.

Varios autores han propuesto que las especies de plantas tropicales se distinguen por su capacidad de regenerar según el tamaño del claro y las condiciones de micrositio (Jones 1956, Schulz 1960, Whitmore 1975, 1983, Pickett 1976, Ricklefs 1977, Hartshorn 1978, 1980, Denslow 1980, Orians 1982, Foster y Brokaw 1982). Estos trabajos identifican las especies que regeneran y alcanzan el dosel después de la caída natural de una rama o un árbol, explicando la regeneración de los bosques tropicales a través de varias teorías como la de densidad y sobrevivencia (Janzen 1970, Connell et al. 1984), la de mosaico de claros (Denslow 1987), la especialización de nichos (Grubb 1977) y la especialización de gremios (Hubbell y Foster 1986). En general, estos trabajos concluyen que los claros son un requisito necesario para la regeneración de bosques.
Numerosos trabajos han propuesto que las especies vegetales pueden diferenciarse de acuerdo a su respuesta ante la presencia de claros (Budowski 1965, Denslow 1980, Hartshorn 1980, Foster y Brokaw 1982, Whitmore 1982, Brokaw 1985). Este punto de vista fomentó una terminología en la dinámica de bosques donde en un extremo se colocaron las especies "pioneras", que dependen de condiciones de alta luz en sitios perturbados o en claros naturales muy grandes, de crecimiento muy rápido, de vida corta, con producción de numerosas semillas pequeñas (Whitmore 1983). Por otro lado, están los árboles no pioneros del bosque maduro, que muestran diversos niveles de tolerancia a la luz, que pueden crecer bajo el dosel del bosque (Uhl 1989, Lieberman et al. 1985, Schupp 1989). Sin embargo, se ha visto que el comportamiento 
de las especies no se ajusta a esta dicotomía (Clark y Clark 1987, 1992).

El reconocimiento de sólo dos estrategias de regeneración de los bosques tropicales, ha servido de base para el desarrollo de las prácticas de manejo silvicultural. Es decir, existen criterios para el manejo de especies tolerantes o intolerantes a claros. Los criterios técnicos para el manejo de las especies forestales dependen principalmente del tamaño del sitio, la frecuencia y la abundancia de la especie de interés, su área basal y el volumen de madera por especie por finca. Namkoong et al. (1996) señalaron que, las prácticas de aprovechamiento forestal causan impacto directamente en el recurso genético de las especies comerciales aserrables y las no aserrables. Según estos autores, se afecta la composición y diversidad vegetal, la edad, la distribución de la densidad y existe un alto riesgo de deriva genética en las poblaciones raras o reducidas. Por estas razones, la necesidad de estudios autoecológicos que brinden mayor información sobre la distribución natural de las especies de interés comercial, las zonas de vida o tipos de bosque donde crecen, la estructura horizontal y vertical de las poblaciones en el bosque, la biología de la reproducción, comportamiento sucesional, es cada vez mayor. Esto por cuanto estas representan un grupo de especies sobre las que se realiza una extracción selectiva y dirigida, y de las que se desconoce casi toda su biología, excepto el valor de su madera.

Los objetivos fueron: (1) Determinar la distribución de los árboles de nazareno (Peltogyne purpurea) en categoría diamétricas, en un bosque intervenido de la Península de Osa. (2) Determinar el radio de dispersión de la regeneración del nazareno alrededor de los árboles madre. (3) Cuantificar el número de individuos por categoría de plántula, brinzal, latizal y fustal de la población de nazareno.

\section{MATERIALES Y MÉTODOS}

Especie estudiada: El género Peltogyne (Fabaceae) tiene una distribución geográfica que corresponde a América del Sur Tropical, (Chichignoud et al. 1990, Nascimento y Proctor 1997). Sin embargo, la especie estudiada en este trabajo, P. purpurea Pittier, (FabaceaeCaes) es un árbol nativo de Costa Rica y Panamá (Holdridge y Poveda 1975) donde habita en lomas o áreas bien drenadas, con climas muy húmedos. P. purpurea conocida como nazareno por el color morado de su madera, es una especie de bosque natural, emergente de hasta 30 a $40 \mathrm{~m}$ de alto y en la región de Los Mogos forma tipos de asociaciones donde domina la estructura horizontal. Se caracteriza por ser un árbol de hasta $50 \mathrm{~m}$ de altura, presenta grandes gambas delgadas, corteza gris y lisa. Sus hojas tienen un par de hojuelas elípticas, con flores de color blanco, olorosas, pequeñas que se producen en panículas subterminales. Los frutos son legumbres oblicuas, planas, de unos $5 \mathrm{~cm}$ de largo y con una sola semilla. La fructificación no ocurre anualmente (Holdridge y Poveda 1975, Vílchez y Rocha 2004, Rocha et al. 2006).

En Costa Rica, el nazareno es una especie maderable de alto valor comercial. En la actualidad, esta especie se encuentra restringida a unos pocos relictos del Pacífico Sur y Medio de la provincia de Puntarenas, específicamente en: (1) La Reserva Biológica Carara, (2) Zona Protectora la Cangreja-Puriscal, (3) Refugio de Vida Silvestre Golfito, lugares donde es escaso, (4) Península de Osa principalmente en la región de Los Mogos. En los primeros tres sitios mencionados, esta especie es relativamente escasa; sin embargo, en la región de Los Mogos es abundante, y con buena regeneración menor a $50 \mathrm{~cm}$ de alto. Debido a su explotación acelerada, se considera que se encuentra bajo amenaza de extinción en Costa Rica (Jiménez y Poveda 1991).

Ubicación del área de estudio: Se realizó en la Península de Osa, Puntarenas, Costa Rica; específicamente en la región conocida como "Los Mogos", 845' N, 83²2'47” W y una elevación de $150 \mathrm{~m}$ ), de marzo de 1995 hasta diciembre de 1996. Según el mapa de Zonas de Vida de Costa Rica el sitio corresponde a un 
bosque muy húmedo premontano transición a basal (Tosi 1971, Holdridge 1987). La precipitación tiene un ámbito muy amplio que va desde 3000 a $4000 \mathrm{~mm}$ como promedio anual, y presenta un período seco bien definido que tiene una duración muy variable (de 0 a 5 meses). La temperatura promedio anual varía entre 24 y $27^{\circ} \mathrm{C}$. La vegetación natural tiene entre 30 y $40 \mathrm{~m}$ de altura, de tres estratos y es siempreverde, con algunas especies deciduas durante la estación seca (Bolaños y Watson 1993). Esta es una de las regiones con más activa deforestación en el presente (Sader y Joyce 1988, Stiles y Skutch 1989, De Camino 1992, Sánchez 1996).

En este sitio se definieron cuatro parcelas de 1 ha cada una en un bosque sometido a manejo forestal. Se utilizaron cuatro procedimientos para la extracción de la madera cortada: (1) método tradicional, (2) método mejorado, (3) con el uso de bueyes y tractor, (4) únicamente con el uso de bueyes. El método mejorado difiere del tradicional en que en él se controla la dirección de caída del árbol, se limita la operación del tractor y se reduce el tamaño del área de impacto del aprovechamiento. La intensidad de corta fue la misma en todos los tratamientos, sólo se cortaron de 3-4 árboles con diámetros mayores a $60 \mathrm{~cm}$ por hectárea. Las parcelas de 1 ha se ubicaron dentro de cada uno de estos cuatro tratamientos, se dividieron en subparcelas de 10 x 10 m, y se identificaron y marcaron todos los individuos mayores a $10 \mathrm{~cm}$ de diámetro. Se analizó la distribución diamétrica de la población de los fustales.

Categorías de regeneración: Se utilizó la metodología de Wyatt-Smith (1962) modificada, citados por Saénz y Finegan (2000). Para ellos se anotó el número de individuos presentes en cada sub-parcela, según las siguientes categorías de regeneración: (1) plántulas: todos aquellos individuos que se encontraron hasta la aparición de las primeras hojas verdaderas (eófilas), (2) brinzales: todas las plantas que estaban entre la aparición de las primeras hojas verdaderas y $1.5 \mathrm{~m}$ de altura, (3) latizales: individuos mayores de $1.5 \mathrm{~m}$ de altura y diámetros a la altura del pecho hasta $9.9 \mathrm{~cm}$, (4) fustales: todos aquellos individuos con un diámetro a la altura del pecho mayor a $9.9 \mathrm{~cm}$. Todos fueron marcados y su supervivencia se evaluó por un período de dos años.

Distribución espacial de las plántulas y brinzales: El patrón de dispersión plántulas y brinzales a partir de los árboles productores de semillas, se determinó utilizando el $50 \%$ de los individuos adultos y emergentes en pie de P. purpurea de cada hectárea. Se trató que los árboles seleccionados estuvieran distanciados entre sí para evitar traslape entre las áreas de muestreo. En total, se estudiaron 11 árboles en las 4 ha analizadas. Desde cada árbol se realizaron transectos en los cuatro puntos cardinales, donde cada uno tuvo una longitud de $22 \mathrm{~m}$ y se subdividieron en 11 subparcelas: (a) dos subparcelas de $2 \mathrm{~m}$ de longitud por $2 \mathrm{~m}$ de ancho, (b) tres subparcelas de $2 \mathrm{~m}$ de largo por $2.5 \mathrm{~m}$ de ancho, (c) tres de $2 \mathrm{~m}$ de longitud $\mathrm{x}$ $3 \mathrm{~m}$ de ancho y (d) tres de $2 \mathrm{~m}$ de largo $\mathrm{x} 4 \mathrm{~m}$ de ancho (Fig. 1). Se utilizó esta estrategia con el fin de reducir el sesgo de una intensidad de muestreo mayor a la orilla del árbol que en la región exterior del mismo e intensificar el muestreo en las áreas donde se espera que

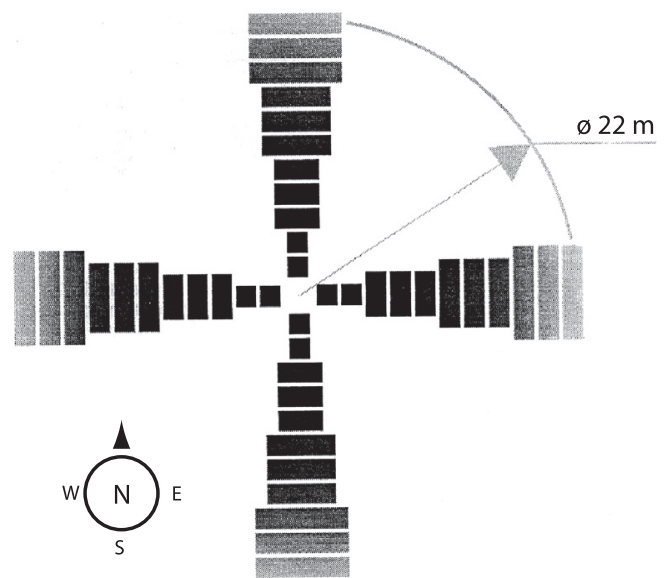

Fig. 1. Ubicación de las subparcelas a lo largo de los transectos para el estudio de la dispersión de plántulas, brinzales y latizales a partir de árboles madre.

Fig. 1. Location of subplots along the sampling transects for the study of the number seedlings, saplings and juveniles found around maternal trees. 
haya mayor incidencia de jóvenes. De un área de $15205308 \mathrm{~m}^{2}$, se muestrearon $65 \mathrm{~m}^{2}$ por transecto para un total de $260 \mathrm{~m}^{2}$ por árbol, lo que implica una intensidad de muestreo de un $17.1 \%$ por árbol, en las 4 ha el muestreo fue de $7.15 \%$.

Datos de crecimiento de plántulas y brinzales: Se marcaron 40 individuos de la categoría de brinzal porque no se encontraron plántulas; se midieron semestralmente para observar si pasaban o no a la categoría de latizal. La tasa de crecimiento semestral se calculó tomando la diferencia promedio en la altura de los brinzales entre dos mediciones consecutivas.

\section{RESULTADOS}

Estructura poblacional: El número de árboles de $P$. purpurea mayores o iguales a $10 \mathrm{~cm}$ de diámetro a la altura del pecho (dap), en las 4 ha estudiadas fue de 29 antes de la corta. Se cortaron un total de 12 y se encontró que el número mayor de individuos de $P$. purpurea cortados se concentró en la categoría de $70-80 \mathrm{~cm}$. El ámbito de diámetro entre $10-20 \mathrm{~cm}$, tuvo tres individuos antes de la corta y varió después de ésta (Fig. 2). En la categoría de 20 a $30 \mathrm{~cm}$ no se encontraron árboles. Después de la corta, en la categoría de 50-60 $\mathrm{cm}$ hubo una baja en el número de individuos con respecto a la categoría anterior (40-50 que se mantuvo igual) y en la $60-70 \mathrm{~cm}$ también bajó. Los árboles sobre los diámetros de $80 \mathrm{~cm}$ de dap fueron los que más se cortaron. En la categoría de $90 \mathrm{~cm}$ desapareció el único individuo y entre 90 a 110 se dejó apenas uno. Estos datos demuestran que antes y después del aprovechamiento forestal en estas poblaciones de nazareno siempre han faltado individuos en alguna de las categorías diamétricas. El análisis de la distribución de las categorías diametrales en ambos trabajos, revela que éstas tienen una forma de campana.

Distribución espacial de la regeneración: Los datos del radio de dispersión de las plántulas, brinzales y latizales alrededor de una muestra de 11 individuos productores de semilla se resumen en el cuadro 1. Cabe señalar, que en el muestreo realizado en este estudio no se encontraron individuos en la categoría de plántulas. Se encontró un total de 340 individuos regenerando alrededor de los 11 árboles semilleros, el $98.8 \%$ perteneció a la categoría de brinzal y sólo un $1.47 \%$ a la categoría de latizal. Se puede observar en la columna del

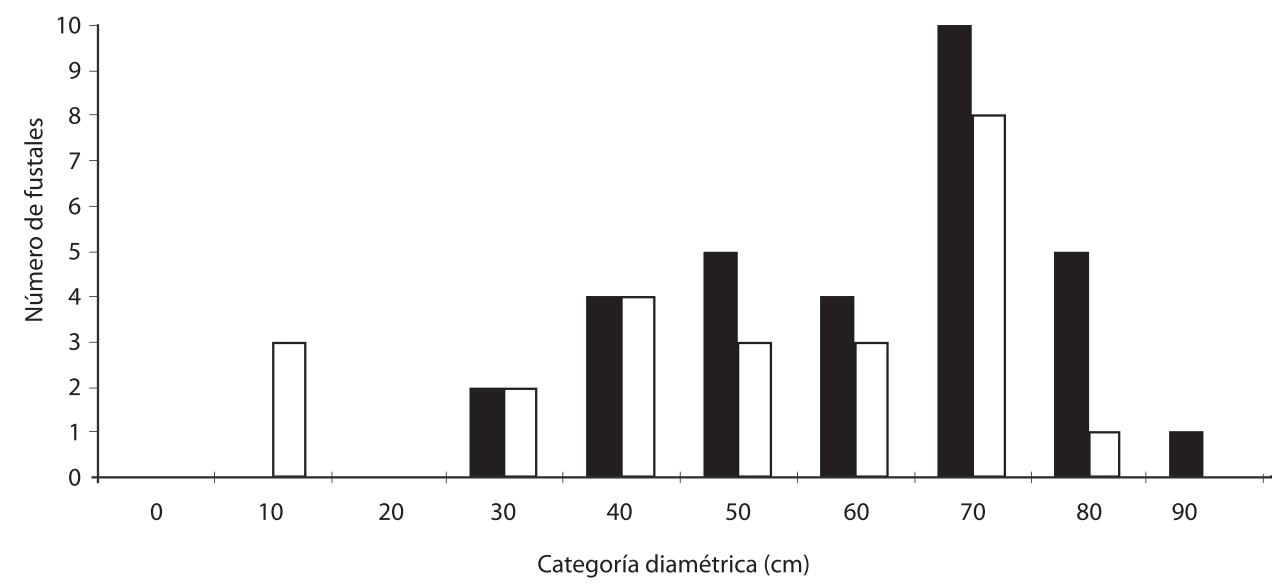

Fig. 2. Número de fustales de nazareno (Peltogyne purpurea) encontrados por categoría diamétrica antes (negro) y después (blanco) de la corta en Los Mogos, Península de Osa, Puntarenas, Costa Rica.

Fig. 2. Number of juvenile trees of nazareno (Peltogyne purpurea) in each diametric class before (black) and after (white) logging in Los Mogos, Península de Osa, Puntarenas, Costa Rica. 
total por árbol que la desviación estandard es muy parecida al promedio. Esto significa que, existe una alta variación en la cantidad de regeneración alrededor de los árboles semilleros o madres, se puede decir que, con los 11 árboles muestreados hay un promedio de 31 individuos regenerando a su alrededor, pero esta cantidad puede oscilar entre 4 y 59 .

La figura 3 muestra el número de individuos de brinzales de P. purpurea por $\mathrm{m}^{2}$ a diferentes distancias al árbol madre. Esta figura demuestra que la mayoría de la regeneración encontrada está en los primeros cuatro metros alrededor del árbol, luego decae y vuelve a tener otro pico al final de la copa del árbol. Sin embargo, no se observaron diferencias significativas entre número de individuos por distancia.

\section{DISCUSIÓN}

Los resultados obtenidos en este estudio demuestran que, en $P$. purpurea, no se presentan individuos en todas las categorías diametrales consideradas. Su forma de distribución en "forma de campana" es característica de varias especies con problemas de regeneración y de alto valor comercial (Rollet 1971, 1980, Lamprecht 1990). Esta forma de la distribución de los individuos, que no se ajusta a la "J" invertida propuesta por los modelos silviculturales de manejo sostenible de los bosques tropicales (Lamprecht 1990, Howard y Valerio 1992), trae como consecuencia que, no se encuentren plántulas en los muestreos realizados y que la mayoría de los individuos encontrados se incluyeran en la categoría de brinzales, pues estos tienen una alta supervivencia, aunque su crecimiento es muy limitado.

Otros autores han estudiado la estructura poblacional de árboles tropicales (Henriquez y Soussa 1989, Korning y Balslev 1994, Nascimiento y Proctor 1997). Por ejemplo, Nascimento y Proctor (1997) comunicaron que en bosques dominados por Peltogyne gracilipes, la mayoría de los individuos de esta

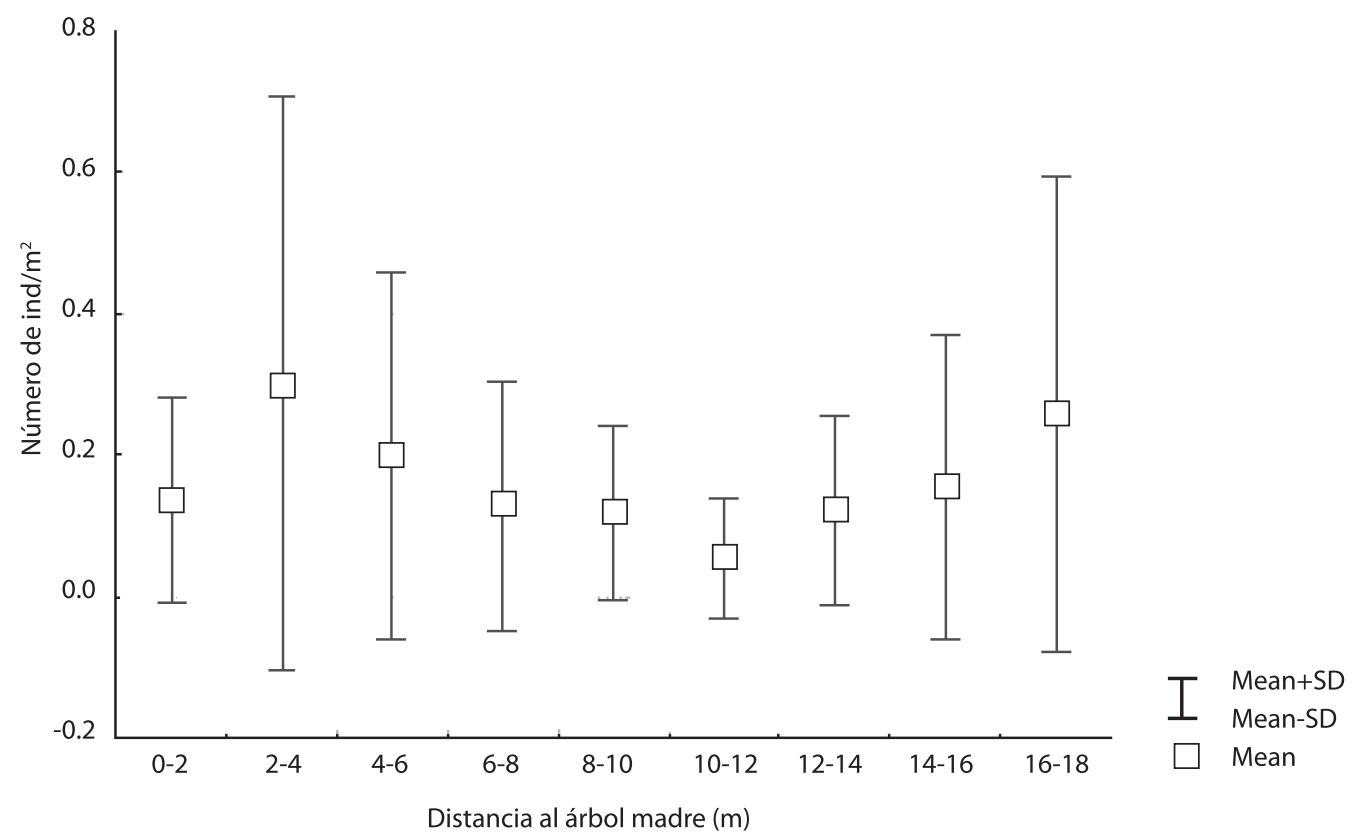

Fig. 3. Análisis de variancia sobre la distribución de los brinzales de Peltogyne purpurea alrededor de los árboles madre en un bosque intervenido por el aprovechamiento forestal en la Península de Osa, Costa Rica.

Fig 3. Analysis of variance for the distribution of saplings around maternal trees in a logged forest in Los Mogos, Península de Osa, Puntarenas, Costa Rica. 
CUADRO 1

Identificación del árbol y número de plántulas, brinzales y latizales encontrados alrededor de 11 árboles semilleros en la Penínisula de Osa, Costa Rica

TABLE 1

Adult tree identification and number of seedlings, saplings, and juveniles trees found around each of the 11 maternal trees in Los Mogos, Península de Osa, Costa Rica

$\begin{array}{ccccc}\text { Árbol } & \text { Plántulas } & \text { Brinzal } & \text { Latizal } & \begin{array}{c}\text { Total } \\ \text { por árbol }\end{array} \\ 88 / 1 & 0 & 75 & 2 & 77 \\ 67 / 1 & 0 & 8 & 1 & 9 \\ 66 / 1 & 0 & 6 & 0 & 6 \\ 58 / 1 & 0 & 16 & 0 & 16 \\ 49 / 1 & 0 & 2 & 1 & 2 \\ 32 / 2 & 0 & 15 & 0 & 15 \\ 83 / 1 & 0 & 17 & 1 & 18 \\ 7 / 1 & 0 & 62 & 0 & 62 \\ 4 / 1 & 0 & 34 & 0 & 34 \\ 92 / 1 & 0 & 28 & 0 & 28 \\ 42 / 2 & 0 & 73 & 0 & 73 \\ \text { Total } & 0 & 336 & 5 & 340 \\ \text { Prom } & 0 & 30.54 & 0.45 & 30.90 \\ \text { Desv st } & 0 & 27.11 & 0.68 & 27.31 \\ \text { Coef. Var } & 0 & 89.35 & 151.1 & 88.38\end{array}$

especie no alcanzan $\operatorname{los} 10 \mathrm{~cm}$ de diámetro a la altura del pecho (plántulas, brinzales y latizales). Ellos concluyen que este número alto de individuos en las categorías de menor tamaño indican que $P$. gracilipes regenera muy bien en estos bosques. En otro estudio, De Steven (1994) encontró que Quararibea asterolepis, Tetragastris panamensis y Trichilia tuberculata, especies tolerantes a la sombra, la abundancia de individuos jóvenes era muy variable. En $Q$. asterolepis existe un escaso número de plántulas y casi ningún brinzal o latizal (más de $50 \mathrm{~cm}$ de altura). Por otro lado, en $T$. panamensis, se encontró la mayor abundancia de plántulas y brinzales, mientras que en T. tuberculata, existe un número intermedio de individuos en esas mismas categorías. Además, se encontró que la supervivencia de individuos jóvenes aumentaba con el aumento de su tamaño, y que la apertura del dosel era importante para el reclutamiento de estas especies. Estos resultados ilustran diferentes estrategias utilizadas por especies de árboles tropicales, que permiten que sus poblaciones se mantengan en estos ecosistemas.

En este estudio se encontró que los brinzales de $P$. purpurea, no sólo son muy abundantes, sino que permanecen en el bosque y muestran muy poco crecimiento. En los dos años de trabajo se observó que de los 70 brinzales marcados ninguno pasó a la categoría de latizal. La supervivencia de brinzales es similar a lo informado por Nascimento y Proctor (1997) en bosques dominados por $P$. gracilipes. Lo que sugiere que esa alta persistencia parece ser característica del género. Clark y Clark (1987) encontraron que hay individuos que permanecen en los estratos inferiores del bosque fisiológicamente vivos, pero que después de un tiempo no tienen la capacidad de crecer y se convierten en seres ecológicamente muertos. Los resultados con P. purpurea describen que las plántulas de esta especie no regeneran satisfactoriamente en los claros, a pesar de su alta persistencia. El crecimiento de los brinzales fue muy lento, un par de hojuelas por año, que ocurrió con la entrada de la época seca (observación personal), lo que muestra que en las categorías menores a $1.5 \mathrm{~m}$ de alto tiene problemas de crecimiento. Se hace necesario averiguar la edad óptima que debe tener un individuo joven protegido de la luz directa del claro para tener luego la capacidad de crecer, pues se observó que después del aprovechamiento forestal hubo un paso de tres latizales a fustales jóvenes entre 10 y $20 \mathrm{~cm}$ de dap.

La abundancia de $P$. purpurea en la zona de Los Mogos está relacionada con su área basal y la alta supervivencia de brinzales reportada en este estudio. En la estructura vertical hay un alto porcentaje de brinzales, menor de latizales y muy bajo de árboles cuyos diámetros se encuentran entre los 10 y $20 \mathrm{~cm}$ (Fig. 2). En este estudio se observó, un alto reclutamiento de plántulas a inicios de la época lluviosa, 
muchas de las cuales sobreviven si quedan a la orilla de los claros naturales o creados por los tratamientos de extracción de madera. $\mathrm{La}$ mayor mortalidad de brinzales ocurrió a finales de la época seca. Primack y Lee (1991) encontraron que en Macaranga, una especie pionera, ocurre una alta mortalidad a los 3 años después de la intervención y cero mortalidad durante el cuarto año. La alta mortalidad inicial es probablemente un indicador de que el establecimiento de esta especie se ve afectado por los daños ocasionados por la exposición a condiciones alteradas. En P. purpurea la mortalidad fue grande para los brinzales expuestos al sol y la falta de agua, típicas de los claros provocados por la extracción forestal con tamaños promedios de 400 a $500 \mathrm{~m}^{2}$ de área (obs. pers.). La apertura de claros realizados por el aprovechamiento forestal es muy grande y cambia la estructura vertical y horizontal del bosque y por ello afecta sus procesos naturales (Uhl 1989).

El efecto de la distancia al árbol madre ha sido tema de estudio desde los años treinta (Ridley), citado por Janzen (1970). El modelo más conocido es el de Janzen (1970) y Connell (1971), el cual asume que la alta densidad de semillas y plántulas que tiende a concentrarse cerca de los árboles madre, desaparece por completo después de un período determinado. La supervivencia de los individuos jóvenes aumenta con la distancia a la planta madre. Howe y Smallwood (1982), encontraron que los ellyalos de otras especies pueden colonizar el espacio alrededor de las madres, y de esa forma se puede mantener la alta diversidad que caracteriza a los bosques tropicales. Cintra (1997), probó el modelo de Janzen y Connell para dos especies comunes del Amazonas. Encontró que la depredación de ellyalos de Astrocarium murumuru aumentaba con el número de semillas o la densidad pero no con la distancia del árbol madre; pero en el caso de Dipterix micrantha los resultados fueron inversos. Según, este último autor, la supervivencia depende de factores como la cosecha, el año en que se hace la medición, la edad de la progenie, el tipo de depredador que tenga la especie y más aún de la escala del tiempo. P. purpurea es una especie dominante en la región estudiada, sus semillas caen con mayor abundancia cerca de la copa del árbol y hay una alta supervivencia de los brinzales bajo la sombra. Se observó una mayor concentración de la regeneración en los primeros $4 \mathrm{~m}$ al árbol madre y entre los 16-18 m. Este aumento de la supervivencia al inicio, y cerca del final de los transectos, se puede deber a los efectos de la copa de los árboles madre y a la influencia de los vecinos de la misma especie. La relación del número de individuos por $\mathrm{m}^{2} \mathrm{y}$ distancia al árbol madre no mostró diferencias significativas.

El patrón espacial y la magnitud de reclutamiento depende fuertemente de la distribución de las semillas en el momento de la dispersión (Augspurger 1983, 1984). En general, se ha propuesto que entre más grande es la distancia de desplazamiento de las semillas mayor será la probabilidad de reclutamiento (Augspurger 1983, 1984). Sin embargo, Augspurger y Kelly (1984), encontraron en estudios de especies dispersadas por viento que la mortalidad y enfermedades son 72 veces mayor en individuos que crecen a la sombra que aquellos que crecen a la luz. El acúmulo de reclutamiento en los primeros metros fue descrito por Condit et al. (1992) para muchas especies de Isla Barro Colorado y Burkey (1994) para Brosimun alicastrum (Moraceae) en Los Tuxtlas, México. Sin embargo, lo encontrado por estos autores fue que la mayor supervivencia de la regeneración se encontró en los primeros $10 \mathrm{~m}$. En otro estudio, De Steven (1994) encontró que la densidad de plántulas que crecen bajo la sombra es por lo general alta en especies tolerantes a la sombra; pero ésta es mayor debajo de la copa de los árboles y declina con la distancia. Según Condit et al. (1992), la mayor concentración de individuos encontrados en los primeros $10 \mathrm{~m}$ alrededor de los árboles de $B$. alicastrum fue el producto de la interacción entre la curva de densidad de semillas y el aumento en área, de manera que al aumentar la circunferencia alrededor del árbol madre disminuye la probabilidad de depredación o herbivorismo.

En los bosques de la Península de Osa, la estructura de las poblaciones de $P$. purpurea 
pudo cambiar su forma natural por el aprovechamiento forestal. Además del impacto mismo de la corta de árboles y su caída, la construcción de los caminos para la extracción en los alrededores de los árboles muestreados creó claros muy grandes, donde la luz directa causó una alta mortalidad de plántulas recién germinadas y de brinzales. Por este motivo la regeneración en el centro de esos claros desapareció después de un año. Peres y Baider (1997) en trabajos realizados en Brazil con Bertholletia excelsa (Lecythidaceae), encontraron que en áreas donde se cosechó madera fue virtualmente imposible volver hallar plántulas y latizales, lo cual demuestra que algunas especies no vuelven al sistema si son severamente dañadas o si la perturbación causada por la extracción de madera es frecuente. Garwood (1990) encontró en la Isla de Barro Colorado dos temporadas de diseminación máxima de semillas, pero sólo hay una temporada para su germinación, la que ocurre a comienzos de la estación lluviosa. Las plántulas que germinan y sobreviven durante toda la época de lluvia y logran llegar a la siguiente estación seca, seis a ocho meses después, sufren una alta mortalidad porque sus raíces son poco profundas y la alta intensidad lumínica no les permite soportar la falta de agua. Sin embargo, es importante decir que para $P$. purpurea el número de brinzales marcados y establecidos desde agosto de 1995 fue igual al de marzo de 1996, lo cual parece indicar que los brinzales que quedaron a las orillas de los claros o dentro del dosel pueden sobrevivir hasta el final de la época seca.

Pires (1984) y Muller (1981) concluyeron que el reclutamiento efectivo de plántulas y regeneración en bosques maduros, puede depender de la disponibilidad apropiada de claros ocasionados por árboles o ramas caídas. Asimismo, señalaron que, entre mayor sea el efecto de los eventos que influyen en la dinámica de bosques como tormentas, derrumbes, etc., menor será el reclutamiento, por tanto, es importante destacar que el aprovechamiento forestal puede afectar la estructura vertical de algunas especies vegetales. Namkoong et al. (1996) indicaron que, como resultado de las prácticas del manejo forestal se pueden tener cambios en la variación genética, lo cual podría influir en los procesos básicos de la evolución como: (1) deriva genética, por cambios no direccionales en las frecuencias genotípicas de poblaciones reducidas, (2) selección, por la supervivencia y reproducción de los genotipos favorecidos o viables, (3) migración, por intercambio de genes entre poblaciones con distintas frecuencias genotípicas, (4) apareamiento, por intervención en la recombinación de genes entre generaciones. La corta de especies maderables puede traer efectos de deriva genética, selección directa y afectar además la tasa de apareamiento, tal y como lo señalan Namkoong et al. (1996). En la región de Los Mogos, donde existe una corta selectiva de varias especies maderables, entre ellas $P$. purpurea, el tamaño efectivo de población se podría reducir primero por el aprovechamiento forestal y luego por la caída de hasta cuatro árboles por hectárea que típicamente acompaña la corta selectiva. Esto último cobra aún más importancia si se considera que los bosques de esta zona están muy fragmentados como consecuencia de la agricultura y la ganadería.

Uhl (1989) estudió el impacto de la extracción maderera en la Amazonia brasileña. Su trabajo ilustra el efecto del aprovechamiento forestal con una corta de cuatro, ocho o 10 árboles por hectárea y removiendo entre 30 a 50 $\mathrm{m}^{3}$ de madera por hectárea. Sus datos revelan que aún con una baja intensidad de extracción, el $26 \%$ de todos los árboles con diámetros menores a los $10 \mathrm{~cm}$ fueron dañados, el $12 \%$ perdieron sus copas, el $11 \%$ sufrieron daños en sus raíces superiores por la actividad de los tractores y el $3 \%$ sufrieron daños en su corteza. En general, la cobertura total del dosel fue reducida entre un $45 \%$ y un $80 \%$. En la región de Los Mogos, el volumen total de madera con permiso para ser cortado en el período maderero de 1994 fue de $21009.15 \mathrm{~m}^{3}$ (unos 3820 árboles aproximadamente), de éste total, unos $3523.97 \mathrm{~m}^{3}$ fueron para la corta de P. purpurea, lo que representa aproximadamente unos 705 árboles, es decir un $18.5 \%$ de los árboles con permiso de corta para ese período. Estos 
datos indican que si en el caso del nazareno se cumple lo señalado por Lamprecht (1990), que indicó que la alta remoción de individuos afecta más a los jóvenes, las prácticas madereras actuales pueden restringir de manera importante la regeneración de esta especie. Por lo tanto, todo parece apoyar la idea de que esta especie está amenazada de extinción por los altos volúmenes de madera que están siendo extraídos en esta zona (Jiménez y Poveda 1991).

\section{RESUMEN}

Se estudió la regeneración de una población del árbol Peltogyne purpurea Pittier después de una corta selectiva de madera en el bosque lluvioso tropical de Mogos, Península de Osa, Costa Rica. La distribución de los árboles adultos de $P$. purpurea según el diámetro del tallo a la altura del pecho tiene forma de campana. La categoría con la mayor cantidad de individuos correspondió a los árboles con tallos con diámetros entre 70 y $80 \mathrm{~cm}$. No hubo diferencia significativa en el número de brinzales encontrados en los transectos establecidos siguiendo los cuatro puntos cardinales alrededor de los árboles reproductivos (madre). En general, la mayoría de los brinzales se encontraban en los primeros metros alrededor del árbol madre. Se observó otro pico de abundancia de brinzales a la distancia correspondiente con el final de la copa del árbol. La extracción de madera puede tener un efecto negativo en la regeneración de $P$. purpurea.

Palabras clave: Peltogyne purpurea, árbol tropical, estructura poblacional, regeneración de bosques, dinámica poblacional, supervivencia de brinzales.

\section{REFERENCIAS}

Augspurger, C.K. 1983. Seed dispersal of the tropical tree, Platypodium elegans, and the escape of its seedlings from fungal pathogens. J. Ecol. 71: 759-771.

Augspurger, C.K. 1984. Seedling survival among tropical trees species: interactions of dispersal distance, lightgaps, and pathogens. Ecology 65: 1705-1712.

Augspurger, C.K. \& C.K. Kelly. 1984. Pathogen mortality of tropical tree seedlings: experimental studies of the effects of dispersal distance, seedling density, and light conditions. Oecologia 61: 211-217.

Balslev, H.J., B. Ollgaard \& L.B. Holm-Nielsen. 1987. Composition and structure of adjacent unflooded and floodplain forest in Amazonian Ecuador. Opera Bot. 92: 37-57.
Bolaños, R.A. \& V.C. Watson. 1993. Mapa Ecológico de Costa Rica, según el Sistema de Clasificación de Zonas de Vida del Mundo de L.R. Holdridge. Centro Científico Tropical, San José, Costa Rica. Esc. 1:200000.

Brokaw, N.V.L. 1985. Treefalls, regrowth, and community structure in tropical forests, p. 53-69. In S.T.A. Pickett \& P.S. White. (eds.). The ecology of natural disturbance and patch dynamics. Academic, Nueva York, EEUU.

Budowski, G. 1965. Distribution of tropical American rain forest species in the light of successional processes. Turrialba 15: 40-42.

Burkey, T.V. 1994. Tropical tree species diversity: a test of the Janzen-Connel model. Oecology 97: 533-540.

Castillo, U.M. 1991. Establecimiento de parcelas permanentes de muestreo en Bosque Natural, para evaluar el aprovechamiento forestal, Península de Osa, Costa Rica. Práctica de especialidad presentada para optar por el grado de Ingeniero Forestal. Instituto Tecnológico de Costa Rica, Cartago, Costa Rica. 155 p.

Chichignoud, M., G. Deon, P. Detienne, B. Parant \& P. Vantomme. 1990. Atlas de maderas tropicales de América Latina. Organización Internacional de las Maderas Tropicales, Paris, Francia. 218 p.

Cintra, R. 1997. A test of the Janzen-Connell model with two common tree species in Amazonian Forest. J. Trop. Ecol. 13: 641-658.

Clark, D. \& D. Clark. 1987. Análisis de la regeneración de árboles del dosel en bosque muy húmedo tropical: aspectos teóricos y prácticos. Rev. Biol. Trop. 35 (Supl.1): 41-54.

Clark, D.A. \& D.B. Clark. 1992. Life-history diversity of canopy and emergent trees in a neotropical rain forest. Ecol. Monogr. 62: 315-344.

Condit, R., S.P. Hubbel \& R.B. Foster. 1992. Recruitment near conspecific adults and the maintenance of tree and shrub diversity in a neotropical forest. Am. Nat. 140: 261-286.

Condit, R., S.P. Hubbel \& R.B. Foster. 1992. Short-term dynamics of a Neotropical forest. Change within limits. Bioscience 42: 822-828.

Connel, J.H., J.G. Tracey \& L.J. Webb. 1984. Compensatory recruitment, growth and mortality as factors maintaining rain forest tree diversity. Ecol. Monogr. 54: 141-164.

Curtis, J.T. \& R.P. McIntosch. 1951. An upland forest continuum in the prairie-forest border region of Wisconsin. Ecology 32: 3. 
Dawkins, H.L. 1958. The managment of natural tropical high- forest with special reference to Uganda. Imperial Forestry Institute. University of Oxford, Oxford, Reino Unido. 155 p.

De Camino, R. 1992. Evaluación del impacto ambiental de políticas sobre el ambiente y los recursos naturales. Aspectos conceptuales y análisis del caso de Costa Rica, p. 15-40. In Memoria II Congreso Forestal Nacional, San José, Costa Rica.

Denslow, J.S. 1980. Gap partitioning among tropical rainforest trees. Biotropica 12 (Supl. 1): 47-55.

Denslow, J.S. 1987. Tropical rain forest gaps and tree species diversity. Annu. Rev. Ecol. Syst. 18: 431-451.

Denslow, J.S. \& G.S. Hartshorn. 1994. Tree-fall gap environments and forest dynamic processes, p. 120-127. In L.A. Mc Dade, K.S. Bawa, H.A. Hespenheide \& G.S. Hartshorn. (eds.). La Selva ecology and natural history of a Neotropical Rain Forest. University of Chicago, EEUU.

De Steven, D. 1994. Tropical tree seedling dynamics: recruitment patterns and their population consequences for three canopy species in Panama. J. Trop. Ecol. 10: 369-383.

Foster, R.B. \& N.V.L. Brokaw. 1982. Structure and history of the vegetation of Barro Colorado Island, p. 133-150. In E.G. Leigh Jr., A.S. Rand \& D.M. Windsor (eds.). The ecology of a tropical forest: seasonal rhythms and long-term changes. Smithsonian Institution, Washington, DC, EEUU.

Garwood, N.C. 1990. Ciclo estacional de germinación de semillas en un bosque semicaducifolio tropical. p. 243-255. In E.G. Leigh Jr., A.S. Rand \& D.M. Windsor (eds.). Ecología de un Bosque Tropical Ciclos estacionales y cambios a largo plazo. Smithsonian Tropical Research Institute, Panamá.

Grubb, P.J. 1977. The maintenance of species richness in plant communities: the importance of the regeneration niche. Biol. Rev. 52: 107-145.

Hartshorn, G.S. 1978. Tree falls and tropical forest dynamics, p. 617-638. In P.B. Tomlinson \& M.H Zimmerman (eds.). Tropical Trees as Living Systems. Cambridge University, Cambridge, Reino Unido.

Hartshorn, G.S. 1980. Neotropical forest dynamics. Biotropica 12 (Supl. 1): 23-30.

Holdridge, L.R. 1987. Ecología basada en zonas de vida. IICA, San José, Costa Rica. 216 p

Holdridge, L.R. \& L.J. Poveda. 1975. Árboles de Costa Rica. Centro Científico Tropical, San José, Costa Rica. p. 317.
Howard, A.F. \& J. Valerio. 1992. A diameter class growth model for assessing the sustainability of silvicultural prescriptions in natural tropical forest. Commonwealth Forestr. Rev. 71(3/4): 171-177.

Hubbel, S.P. \& R.B. Foster. 1986. Canopy gaps and the dynamics of a neotropical forest. Plant Ecology. Blackwell Scientific, Oxford, Reino Unido. p. 77-96.

Lamprecht, H. 1990. Silvicultura en los trópicos. Los ecosistemas forestales en los bosques tropicales y sus especies arbóreas-posibilidades y métodos para un aprovechamiento sostenido. Instituto de Silvicultura de Gottingen. Antonio Carrillo (trad.). Cooperación Técnica, República Federal de Alemania. p. 40-84.

Korning, J. \& H. Balslev. 1994. Growth rates and mortality patterns of tropical lowland tree species and the relation to forest structure in Amazonian Ecuador. J. Trop. Ecol. 10: 151-166.

Janzen, D.H. 1970. Herbivores and the number of tree species in tropical forest. Am. Nat. 104: 501-528.

Jiménez, Q. \& L.J. Poveda. 1991. Arboles maderables nativos de Costa Rica. Museo Nacional de Costa Rica, San José, Costa Rica. 32 p.

Jones, E.W. 1956. Ecological Studies on the rainforest of southern Nigeria IV. The plateau forest of the Okumu Forest Reserve. J. Ecol. 43: 564-594.

Lieberman, I.D., R. Peralta \& G.S. Hartshorn. 1985. Mortality patterns and stand turnover rates in a wet tropical forest in Costa Rica. J. Ecol. 73: 915-924.

Muller, C.H. 1981. Castanha-do-Brasil: estudos agronomicos. Embrapa/Cpatu, Documentos 1: 1-25.

Namkoong, G., T. Boyle, H.R. Gregorius, H. Joly, O. Savolainen, W. Ratnam \& A. Young. 1996. Testing Criteria and Indicators for Assessing the Sustainability of Forest Management: Genetic Criteria and Indicators. Center International Forestry Research (CIFOR). $13 \mathrm{p}$

Nascimento, M.T. \& J. Proctor. 1997. Population dynamics of five tree species in a monodominant Peltogyne forest and two other forest types on Maracá Island, Roraima, Brazil. Forest Ecol. Manage. 94: 115-128.

Orians, G.H. 1982. The influence of tree-falls on tropical forest species richness. Trop. Ecol. 23: 255-279.

Peres, C.A. \& C. Baider. 1997. Seed dispersal, spatial distribution and population structure of Brazilnut trees (Bertholletia excelsa in southeastern Amazonia. J. Trop. Ecol. 13: 595-616.

Picket, S.T.A. 1976. Succession: an evolutionary interpretation. Am. Nat. 110: 107-119. 
Pires, J.M. 1984. The Amazonian forest, p. 581-602. In H. Sioli (ed). The Amazons limnology and landscape ecology of a mighty tropical river and its basin. Junk, Dordrecht, Holanda.

Primack, R.B. 1992. Tropical Community Dynamics and Conservation Biology. Bioscience 42(11): 818-821.

Primack, R.B. \& H.S. Lee. 1991. Population dynamics of pioneer (Macaranga) trees and understorey (Mallotus) trees (Euphorbiaceae) in primary and selectively logged Bornean rain forests. J. Trop. Ecol. 7: 439-457.

Ricklefs, R.E. 1977. Environmental heterogeneity and plant species diversity: a hypothesis. Am. Nat. 111: 376-381.

Rocha, O.J., B. Vílchez \& A.L. Araya. 2006. A mast fruiting episode of Peltogyne purpurea (Caesalpinaceae) in the Osa Peninsula, Costa Rica. Rev. Biol. Trop. 52: (in press).

Rollet, B. 1971. La regeneración natural en el bosque denso siempreverde de llanura de la Guayana Venezolana. Boletín IFLA 35: 39-74.

Rollet, B. 1980. Organización. In Anónimo. Ecosistemas de los bosques tropicales: informe sobre el estado de los conocimientos. Roma, Italia. UNESCO-PNUMAFAO: $126-162$

Sader, S. \& A. Joyce. 1988. Deforestation rates and trends in Costa Rica, 1940 to 1983. Biotropica 20: 11-19.

Saénz, G.P. \& B. Finegan. 2000. Monitoreo de la regeneración natural con fines de manejo forestal. Manejo Forestal Tropical 15: 1-8.

Sanchez, G.A. 1996. Assessing Land Use / Cover Change in Costa Rica. Pennsylvania State University, Filadelfia, Pennsylvania, EEUU. 186 p.
Schulz, J.P. 1960. Ecological studies on the rain forest in northern Surinam. Verh. K. Ned. Akad. Wet., Afd. Naturk'd. Tweed Reeks. 53: 1-367.

Schupp, E.W., H.F. Howe, C.K Augspurger \& D.J. Levey. 1989. Arrival and survival in tropical treefall gaps. Ecology 70: 562-564.

Tosi, J. 1971. Mapa ecológico de Costa Rica. Centro Científico Tropical, San José, Costa Rica. Esc. $1: 750000$

Uhl, C. 1989. Ecological Impacts of Selective Logging in the Brazilian Amazon: A Case Study from the Paragominas Region of the State of Pará. Biotropica 21: 98-106.

Vílchez, B.V \& O.J. 2004. Rocha. Fenología y biología reproductiva del nazareno (Peltogyne purpurea Pittier) en un bosque intervenido de la Península de Osa, Costa Rica, América Central. Kurú: Rev. Forest. 1: 1.

Whitmore, T.C. 1974. Change with time and the role of cyclones in tropical rain forest on Kolombangara, Solomon Island. Commonwealth Forestry Institute Paper, 46, Oxford, Reino Unido. 68 p.

Whitmore, T.C. 1975. Tropical Rain Forest of the far East. Clarendon, Oxford, Reino Unido. 260 p.

Whitmore, T.C. 1982. On pattern and process in forest, $\mathrm{p}$. 45-49. In E.I. Newman (ed.). The plant community as a working mechanism. Brit. Ecol. Soc. Special Pub. No. 1, Blackwell Scientific, Oxford, Reino Unido.

Whitmore, T.C. 1983. Secondary succession from seed in tropical forest. Forestry Abstracts 44: 767-779.

Whitmore, T.C. 1990. An Introduction to Tropical Rain Forest. Clarendon, Oxford, Reino Unido. p. 9-35. 
